Research Article

\title{
A Comparative Transcriptomic Analysis of Human Placental Trophoblasts in Response to Pathogenic and Probiotic Enterococcus faecalis Interaction
}

\author{
Qianglai Tan, ${ }^{1,2}$ Zhen Zeng, ${ }^{1}$ Feng $X{ }^{2}{ }^{2}$ and Hua Wei ${ }^{2}{ }^{2}$ \\ ${ }^{1}$ Xiamen Medical College, Xiamen 361023, Fujian, China \\ ${ }^{2}$ State Key Laboratory of Food Science and Technology, Nanchang University, Nanchang 330047, Jiangxi, China \\ Correspondence should be addressed to Hua Wei; weihua@ncu.edu.cn
}

Received 5 October 2020; Revised 17 December 2020; Accepted 12 January 2021; Published 28 January 2021

Academic Editor: Maria De Francesco

Copyright (C) 2021 Qianglai Tan et al. This is an open access article distributed under the Creative Commons Attribution License, which permits unrestricted use, distribution, and reproduction in any medium, provided the original work is properly cited.

With the ability to cross placental barriers in their hosts, strains of Gram-positive Enterococcus faecalis can exhibit either beneficial or harmful properties. However, the mechanisms underlying these effects have yet to be determined. A comparative transcriptomic analysis of human placental trophoblasts in response to pathogenic or probiotic $E$. faecalis was performed in order to investigate the molecular basis of different traits. Results indicated that both E. faecalis Symbioflor 1 and V583 could pass through the placental barrier in vitro with similar levels of invasion ability. In total, 2353 (1369 upregulated and 984 downregulated) and 2351 (1233 upregulated and 1118 downregulated) DEGs were identified in Symbioflor 1 and V583, respectively. Furthermore, 1074 (671 upregulated and 403 downregulated) and 1072 (535 upregulated and 537 downregulated) DEGs were only identified in Symbioflor 1 and V583 treatment groups, respectively. KEGG analysis showed that 6 and 9 signaling pathways were associated with interactions between Symbioflor 1 and V583. GO analysis revealed that these DEGs were mainly related to cellular and metabolic processes and biological regulation. However, 28 and 44 DEGs were classified into terms associated with placental and embryonic development in Symbioflor 1 and V583 treatment groups, respectively. Notably, 9 and 25 unique DEGs were identified only in Symbioflor 1 and V583 treatment groups, respectively. A large proportion of transcriptional responses differed when compared between pathogenic and probiotic E. faecalis interaction, and several unique DEGs and signal pathways were identified in the two different groups. These data enhance our understanding of how different traits can be affected by pathogenic and probiotic E. faecalis and the mechanisms underlying these effects.

\section{Introduction}

Enterococcus faecalis is a Gram-positive bacterium that is commonly found in a variety of different matrices including the alimentary tract and foods. This bacterium has received substantial attention due to the fact that it can exert both beneficial and pathogenic effects [1]. Certain E. faecalis strains are regarded as commensal bacteria or even probiotics for creating a healthy gut environment; however, other strains are considered to be dangerous as they can lead to a variety of nosocomial infections and diseases $[2,3]$. Previous researches have shown that $E$. faecalis is able to pass through biological barriers and can subsequently mediate a variety of effects in the host $[4,5]$. A review by Goldenberg et al. confirmed that various enterococci could transmit to fetus and cause stillbirth [6]. We have previously demonstrated that $E$. faecalis OG1RF can pass through the placental barrier of pregnant mice, translocate into the fetus, and then affect both fetal and placental growth and development [7]. We demonstrated that $E$. faecalis OG1RF induces placental and embryonic development retardation, stress and stimulus response activation, apoptosis, immune response disorder, and cell adhesion disintegration of placental trophoblasts through various signaling pathways using real-time PCR and DNA microarray [8]. E. faecalis has also been identified in meconium obtained from healthy neonates. The presence of this type of bacteria in meconium could initiate gut colonization as an adaptation to the fetal gut to prepare the fetus 
for life outside the mother [9]. Albesharat et al. found that E. faecalis was present in the feces of mothers and their babies and hypothesized that the initial bacterial colonization of the infant occurs via vertical transmission from mothers to infants [10]. The close relationship between two typical E. faecalis strains (Symbioflor 1 [11] and V583 [12]) has been studied in significant detail with regards to human pregnancy. E. faecalis Symbioflor 1 (SymbioPharm, Herborn, Germany) is recognized as a probiotic strain that can alleviate the symptoms of irritable bowel syndrome by improving the microbial balance in the intestine. E. faecalis V583 is a vancomycin-resistant prototype clinical isolate that causes opportunistic nosocomial infection worldwide. Meanwhile, a human placental choriocarcinoma BeWo cell lines, a widely recognized in vitro placental model [13], was adopted. The BeWo cell line is regularly used to study a range of placental functions, including transplacental transport and infection caused by viruses and bacteria. DEGs of BeWo cells associated with $E$. faecalis invasion were identified by using DNA microarray, and transcriptomic profiling was compared by using Gene Ontology (GO) and Kyoto Encyclopedia of Genes and Genomes (KEGG) pathway database [14].

In this study, we first constructed an interaction model between two different E. faecalis strains and BeWo cells. Then, we compared the invasion abilities (adhesion ability and internalization ability) between the two E. faecalis strains. Subsequently, we performed comparative transcriptomic profiling by using a DNA microarray (Illumina Human HT-12 v4 Expression Bead Chip), GO analysis, and KEGG pathway analysis. The main objective of this study was to gain insights into the differences in transcriptional regulation between strains of $E$. faecalis that can exhibit dualistic behavior towards host health. The study was designed to identify the different traits that might be affected by putative pathogenic or probiotic E. faecalis. The identification of such traits is crucial as this information may ultimately contribute to the future development of strategies for the prevention and treatment of invasion and infection caused by different $E$. faecalis strains.

\section{Materials and Methods}

2.1. Bacterial Strains and Cell Lines. The bacterial strains used in this study are listed in Table 1. In brief, strains of E. faecalis strains, clinical pathogen V583 (ATCC 700802), and probiotic Symbioflor 1 (DSM 16431) were cultured in Trypticase soy broth (TSB) at $37^{\circ} \mathrm{C}$ for $24 \mathrm{~h}$ with shaking at $180 \mathrm{rpm}$. The bacteria were then harvested by centrifugation at $6000 \mathrm{rpm}$ for $10 \mathrm{~min}$, washed twice with sterile PBS (0.01M, pH 7.4), and reconstituted in cell culture medium DMEM/F-12 (Solarbio, Beijing, China) to yield a concentration of $10^{9} \mathrm{CFU} / \mathrm{mL}$ before use.

The human choriocarcinoma cell line BeWo was purchased from Action-award Biotech Co., Ltd. (Guangzhou, China) and cultured as described previously [8]. In brief, BeWo cells were cultured in DMEM/F-12 medium supplemented with $10 \%(\mathrm{v} / \mathrm{v})$ FBS (Gibco, Grand Island, NY) at $37^{\circ} \mathrm{C}$ under a $5 \% \mathrm{CO}_{2}$ atmosphere, until approximately $80 \%-$ $90 \%$ cell confluence.

2.2. Invasion Assays. Invasion assays were performed in accordance with our previous study [8]. BeWo cells were transferred into 24-well plates (Corning, NY) and cultured for 24-48 h until a confluent monolayer was obtained. A $5 \mu \mathrm{L}$ aliquot of resuspended bacteria was then added to each well containing $495 \mu \mathrm{L}$ of cell culture medium and incubated for 60 minutes.

The total number of invading bacteria was determined by twice dip-washing with sterile PBS to remove free bacteria and lysing the BeWo cells with $500 \mu \mathrm{L}$ of $0.5 \%$ Triton X-100/ PBS to release the internalized bacteria. Serial dilutions were spread onto Trypticase soy agar (TSA) plates and incubated at $37^{\circ} \mathrm{C}$ overnight. The numbers of internalized bacteria were then determined by adding $100 \mu \mathrm{g} / \mathrm{mL}$ of gentamicin and $50 \mu \mathrm{g} / \mathrm{mL}$ of penicillin and incubating for $60 \mathrm{~min}$ to kill any viable extracellular bacteria that were still present. The cells were then washed twice, lysed, and the bacterial count determined as described above. $P$ values were calculated using Student's $t$-test.

2.3. DNA Microarray and Data Analysis. Incubation experiments were performed as described in our previous study [8]. In brief, BeWo cells were grown in 24-well plates and cultured for $24-48 \mathrm{~h}$ until $80 \%-90 \%$ confluency. E. faecalis V583 and Symbioflor 1 were then harvested and resuspended in DMEM/F-12 cell culture medium to $10^{9} \mathrm{CFU} / \mathrm{mL}$. A $5 \mu \mathrm{L}$ aliquot of resuspended E. faecalis V583 and Symbioflor 1 was then added to each well containing $495 \mu \mathrm{L}$ of cell culture medium and incubated for $4 \mathrm{~h}$. The same amount of cell culture medium, but without any E. faecalis strains, was used as a negative control. Total RNA was extracted using TRNzol Total RNA Reagent (TIANGEN, Beijing, China). DNA microarrays were performed by Beijing EMTD Technology Development Co., Ltd. using an Illumina Human HT-12 v4 Expression BeadChip system (Illumina, Inc., San Diego, CA). In brief, RNA was adjusted to a concentration of $200 \mathrm{ng} / \mu \mathrm{L}$, followed by first and then second strand cDNA synthesis. Double-stranded cDNA was then purified with a filter cartridge, and cRNA was synthesized by T7 RNA polymerase transcription in vitro. Following purification and quantification, the cRNA was hybridized with the BeadChip, washed, scanned, and analyzed. Illumina expression data were deposited in the NCBI Gene Expression Omnibus (GEO) database under the accession number GSE75626. DEGs were analyzed using GO and KEGG pathway databases.

2.4. Statistical Analysis. Unless specified, all experiments were performed in triplicate. All data were analyzed using statistics programs contained in SigmaPlot 11.0 (Systat Software, San Jose, CA). 
TABLE 1: Enterococcus faecalis strains used in this study.

\begin{tabular}{|c|c|c|c|c|c|c|}
\hline Strain & Number & Country & Source & Isolation & Characteristics & References \\
\hline Symbioflor 1 & DSM 16431 & Germany & Nonhospitalized person & Feces & Probiotic & [11] \\
\hline V583 & ATCC 700802 & USA & Hospitalized patient & Blood & Pathogen, Ery ${ }^{\mathrm{R}}$, Gen ${ }^{\mathrm{R}}$, and $\operatorname{Van}^{\mathrm{R}}$ & {$[12]$} \\
\hline
\end{tabular}

*Ery, erythromycin; Gen, gentamicin; Van, vancomycin; R, resistance.

\section{Results}

3.1. Invasion Ability of E. faecalis. The number of E. faecalis strains present in BeWo cells was determined using invasion assays. As shown in Figure 1(a), $8.42 \pm 0.13 \log _{10} \mathrm{CFU} / \mathrm{mL}$ of Symbioflor 1 and $8.66 \pm 0.18 \log _{10} \mathrm{CFU} / \mathrm{mL}$ of V583 were identified in BeWo cells after incubation under the same conditions. These results showed that incubation conditions did not cause any deviations in the detection of invasion ability.

As demonstrated in Figure 1(b), the invaded cell counts of Symbioflor 1 and V583 strains were $6.76 \pm 0.20$ and $6.86 \pm 0.20 \log _{10} \mathrm{CFU} / \mathrm{mL}$, respectively, while those of internalized Symbioflor 1 and V583 were $2.48 \pm 0.35$ and $2.06 \pm 0.50 \log _{10} \mathrm{CFU} / \mathrm{mL}$, respectively (Figure 1(c)). These results showed that there was no significant difference in the in vitro invasion ability when compared between the two strains $(P>0.05)$. These data indicated that both the pathogenic and probiotic E. faecalis strains could pass through the placental barrier. Similar observations were noted with regards to the in vitro invasion ability when compared between the two strains.

3.2. Differential Gene Expression Profile Analysis. DNA microarray techniques were used to compare the gene expression patterns of untreated BeWo cells with those infected by E. faecalis. After applying cutoffs for induction (ratio $>2.0$-fold) and suppression (ratio $<0.5$-fold), out of a total of 47,231 genes on the BeadChip, a total of 2353 DEGs, including 1369 upregulated genes and 984 downregulated genes were identified in the Symbioflor 1 treatment group. In the V583 treatment group, a total of 2351 DEGs, including 1233 upregulated genes and 1118 downregulated genes, were identified. Furthermore, 1279 of the total number of DEGs were found to be common to both treatment groups, thus accounting for $54.36 \%$ and $54.40 \%$ of the DEGs in Symbioflor 1 and V583 treatment groups, respectively (Figure 2(a), Tables S1 and S2).

In addition, 698 of the total number of upregulated DEGs were found to be common to both strains, thus accounting for $50.99 \%$ and $56.61 \%$ of the DEGs in the Symbioflor 1 and V583 treatment groups, respectively (Figure 2(b)). Furthermore, 581 of the downregulated DEGs were found to be common to both strains, thus accounting for $59.04 \%$ and $51.97 \%$ of the DEGs in the Symbioflor 1 and V583 treatment groups, respectively (Figure 2(c)).

In general, both pathogenic and probiotic E. faecalis treatment groups showed a similar number of total DEGs. However, the number of upregulated and downregulated genes varied significantly in both groups. Hence, DEGs were then mapped using the KEGG database for signal pathway analysis to gain further understanding of their biological function.

3.3. Signal Pathway Analysis of DEGs. According to signal pathway analysis of DEGs, six main terms associated with BeWo cells in response to invasion by Symbioflor 1, including the MAPK signaling pathway, Jak-STAT signaling pathway, adherens junction, $\mathrm{T}$ cell receptor signaling pathway, p53 signaling pathway, and pathogenic Escherichia coli infection. For the V583 treatment group, nine main terms were associated with the response of BeWo cells to invasion, including the MAPK signaling pathway, leukocyte transendothelial migration, p53 signaling pathway, $\mathrm{T}$ cell receptor signaling pathway, apoptosis, ErbB signaling pathway, adherens junction, B cell receptor signaling pathway, and pathogenic Escherichia coli infection. According to the results obtained as shown in Table 2, five common terms were identified in both treatment groups. However, the Jak-STAT signaling pathway in particular was only observed in the Symbioflor 1 treatment group, whereas the ErbB signaling pathway, apoptosis, B cell receptor signaling pathway, and leukocyte transendothelial migration were all identified in the V583 treatment group.

3.4. Bioinformatic Analysis of DEGs. DEGs were characterized functionally by comparison against GO database and classified into three different categories, namely, the biological process, cellular component, and molecular function. For the Symbioflor 1 treatment group, DEGs were categorized into 293 terms in the biological process, 58 terms in cellular component, and 70 terms in molecular function (Table S3). For the V583 treatment group, the DEGs were categorized into 267 terms in the biological process, 59 terms in cellular component, and 62 terms in molecular function (Table S4). According to the comparative analysis of relevant data from both treatment groups, the top three genes were classified under the GO biological process categories related to the same terms used for the cellular process, metabolic process, and biological regulation (Tables S3 and S4).

In particular, for the Symbioflor 1 treatment group, a total of 28 genes were classified into terms associated with placental and embryonic development: placental development, embryonic placental development, in utero embryonic development, and the embryonic process involved in female pregnancy (Table 3). However, for the V583 treatment group, a total of 44 genes were classified into terms associated with placental and embryonic development, namely, placental development, chordate embryonic development, in utero embryonic development, embryonic development ending in birth or egg hatching, embryonic cranial skeleton morphogenesis, and the embryonic process 


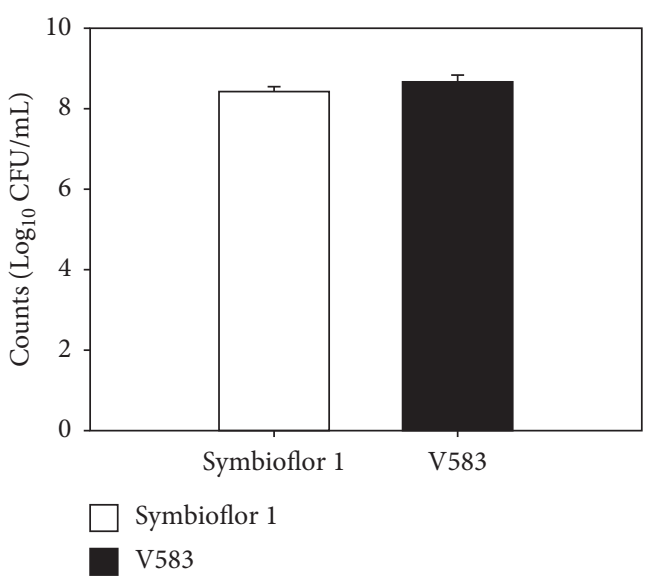

(a)

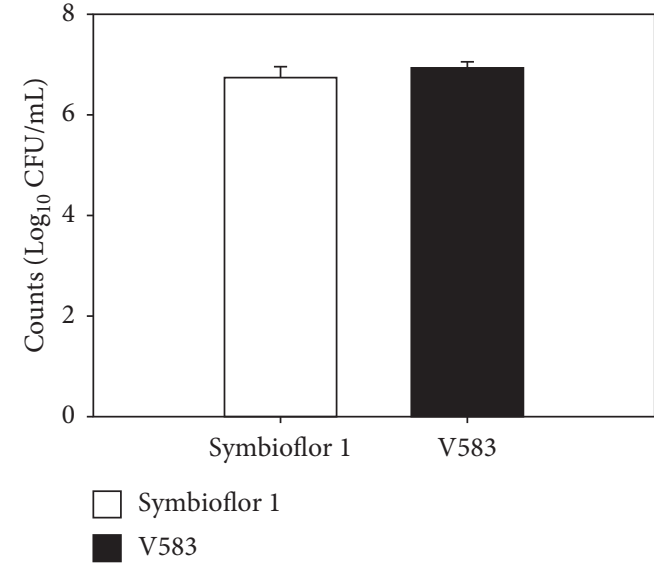

(b)

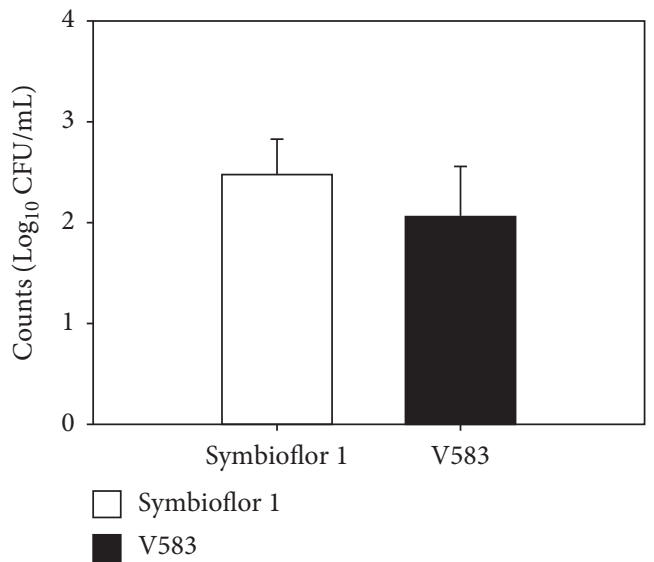

(c)

Figure 1: Invasion ability of E. faecalis strains associated with BeWo cells. (a) Colony forming units (CFUs) of E. faecalis strains after incubation; (b) CFUs of invaded E. faecalis; (c) CFUs of internalized E. faecalis. Values represent mean \pm SD. $P$ values were calculated using Student's $t$-test.

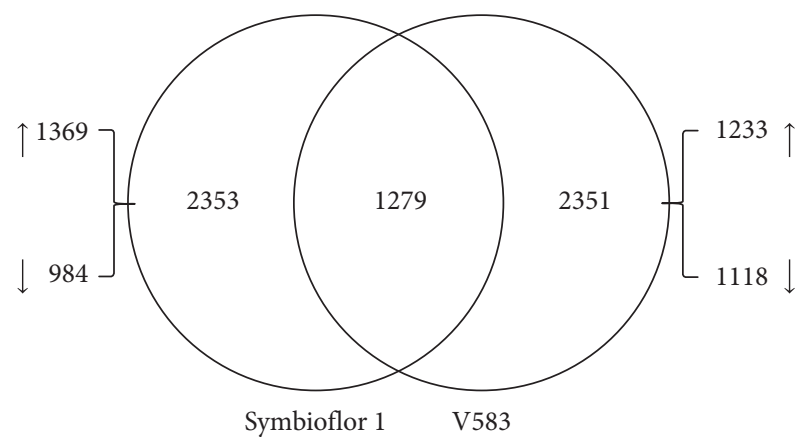

(a)

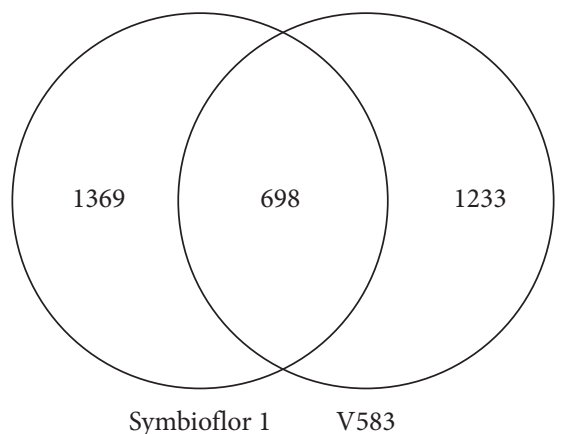

(b)

Figure 2: Continued. 


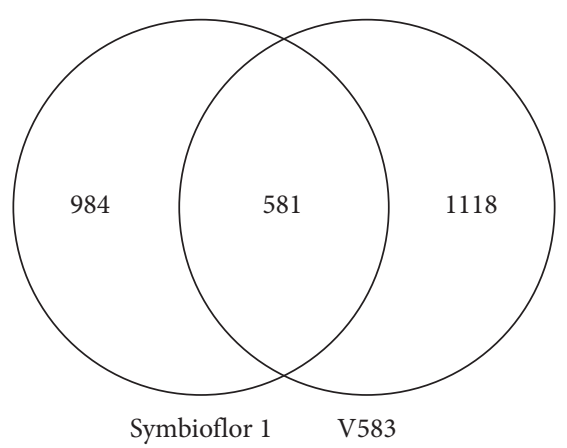

(c)

FIGURE 2: Distribution of DEGs of BeWo cells in response to different E. faecalis strains. Venn diagram showing the number of unique and common DEGs in BeWo cells in response to Symbioflor 1 and V583 infection. (a) Total DEGs; (b) upregulated genes; (c) downregulated genes.

TABLE 2: DEGs containing relevant terms of the signal pathway generated by KEGG pathway analysis.

\begin{tabular}{|c|c|c|}
\hline Group & Term & Genes \\
\hline \multirow{6}{*}{$\begin{array}{l}\text { Symbioflor } 1 \\
\text { group }\end{array}$} & $\begin{array}{l}\text { hsa04010: MAPK signaling } \\
\text { pathway }\end{array}$ & $\begin{array}{c}\text { FGFR4, PDGFB, GNA12, PPM1A, MKNK2, PPM1B, MAP3K7, MAX, FOS, } \\
\text { MAP3K5, MAP3K3, HSPA7, MAP2K7, MYC, HSPA8, RASA2, EGFR, TAOK1, } \\
\text { TGFBR2, DUSP5, ATF4, RPS6KA4, MAPK13, MAPK14, JUN, RAP1A, PLA2G6, } \\
\text { MAPK7, GADD45B, GADD45A, PLA2G2D (31) }\end{array}$ \\
\hline & $\begin{array}{l}\text { hsa04630: Jak-STAT signaling } \\
\text { pathway }\end{array}$ & $\begin{array}{l}\text { PTPN6, CRLF2, LEPR, CBL, SOCS4, BCL2L1, SOCS5, IL7R, IL10, STAT3, LEP, } \\
\text { GH2, ZFP91, STAT4, SPRY1, EP300, IL10RB, IL5RA, SPRED1, MPL, MYC, } \\
\text { PIK3R1 (22) }\end{array}$ \\
\hline & hsa04520: adherens junction & $\begin{array}{l}\text { EGFR, LOC646821, PTPN6, BAIAP2, TGFBR2, CTNND1, ACP1, WAS, CTNNB1, } \\
\text { VCL, MAP3K7, PVRL4, EP300, PVRL1, SORBS1, PVRL2 (16) }\end{array}$ \\
\hline & $\begin{array}{l}\text { hsa04660: T cell receptor signaling } \\
\text { pathway }\end{array}$ & $\begin{array}{l}\text { PTPN6, NFKBIB, CBL, MALT1, IL10, MAP3K7, FOS, NCK2, MAPK13, MAPK14, } \\
\text { PAK4, JUN, ZAP70, MAP2K7, PIK3R1 (15) }\end{array}$ \\
\hline & hsa04115: p53 signaling pathway & $\begin{array}{c}\text { ZMAT3, RPRM, SESN2, CCNG2, CDK2, CCNB1, PPM1D, CDKN1A, CDKN2A, } \\
\text { BAX, RRM2, GADD45B, GADD45A (13) }\end{array}$ \\
\hline & $\begin{array}{l}\text { hsa05130: pathogenic Escherichia } \\
\text { coli infection }\end{array}$ & $\begin{array}{l}\text { LOC646821, NCK2, YWHAZ, KRT18, LOC399942, TUBA3E, WAS, ITGB1, } \\
\text { CTNNB1, TTLL3 (10) }\end{array}$ \\
\hline \multirow{9}{*}{ V583 group } & $\begin{array}{l}\text { hsa04010: MAPK signaling } \\
\text { pathway }\end{array}$ & $\begin{array}{l}\text { FGFR1, FGF18, PDGFB, MRAS, PPP3R1, PPM1A, MKNK2, CACNB3, GNG12, } \\
\text { PPM1B, NFKB2, FOS, MAP3K5, CASP3, NFATC4, MYC, HSPA8, RASA2, EGFR, } \\
\text { NTF4, TAOK1, TGFBR2, DUSP5, ATF4, DUSP2, JUN, GADD45G, RAP1A, } \\
\text { MAPK9, GADD45B, PLA2G3, PLA2G2D, GADD45A (33) }\end{array}$ \\
\hline & $\begin{array}{l}\text { hsa04670: leukocyte } \\
\text { transendothelial migration }\end{array}$ & $\begin{array}{l}\text { ACTB, F11R, LOC646821, NCF4, SIPA1, CTNND1, ITGB1, ITGAM, CTNNB1, } \\
\text { VCL, PTK2, CYBB, PTK2B, RAP1A, PIK3R3, PIK3R1, LOC284620 (17) }\end{array}$ \\
\hline & hsa04115: p53 signaling pathway & $\begin{array}{l}\text { ZMAT3, SESN2, CCNG2, CDK2, CCNE2, PPM1D, CDKN1A, CASP3, CDKN2A, } \\
\text { RRM2, GADD45G, BAI1, APAF1, MDM4, GADD45B, GADD45A (16) }\end{array}$ \\
\hline & $\begin{array}{l}\text { hsa04660: T cell receptor signaling } \\
\text { pathway }\end{array}$ & $\begin{array}{l}\text { BCL10, PTPN6, NFKBIE, NFKBIB, CBL, PPP3R1, IL10, FOS, NCK2, JUN, PAK4, } \\
\text { MAPK9, NFATC4, PIK3R3, PIK3R1, NFATC1 (16) }\end{array}$ \\
\hline & hsa04210: apoptosis & $\begin{array}{l}\text { IAP, AIFM1, PPP3R1, BAD, BCL2L1, CAPN2, CASP6, TNFSF10, CASP3, RIPK1, } \\
\text { PRKAR1B, IL1RAP, APAF1, PIK3R3, PIK3R1 (15) }\end{array}$ \\
\hline & hsa04012: ErbB signaling pathway & $\begin{array}{l}\text { EGFR, CBL, BAD, NCK2, PTK2, CDKN1A, CDKN1B, PAK4, JUN, GAB1, MAPK9, } \\
\text { PIK3R3, MYC, PIK3R1 (14) }\end{array}$ \\
\hline & hsa04520: adherens junction & $\begin{array}{l}\text { ACTB, EGFR, LOC646821, FGFR1, PTPN6, TGFBR2, CTNND1, ACP1, VCL, } \\
\text { CTNNB1, PVRL4, EP300, PVRL1, PVRL2 (14) }\end{array}$ \\
\hline & $\begin{array}{l}\text { hsa04662: B cell receptor signaling } \\
\text { pathway }\end{array}$ & $\begin{array}{l}\text { BCL10, PTPN6, IFITM1, NFKBIE, NFKBIB, PPP3R1, FOS, JUN, NFATC4, } \\
\text { PIK3R3, PIK3R1, BLNK, NFATC1 (13) }\end{array}$ \\
\hline & $\begin{array}{l}\text { hsa05130: pathogenic Escherichia } \\
\text { coli infection }\end{array}$ & $\begin{array}{l}\text { ACTB, LOC646821, NCK2, KRT18, ARPC3, LOC399942, TUBA3E, ITGB1, } \\
\text { CTNNB1, TTLL3 (10) }\end{array}$ \\
\hline
\end{tabular}

involved in female pregnancy. According to the results obtained (Table 3), 19 DEGs were found to be common to both treatment groups.

In addition, the fold-changes for these DEGs are shown in Figure 3. For the Symbioflor 1 treatment group, eight out of nine unique DEGs were upregulated, while one gene (MSX1) was downregulated. For the V583 treatment group, 10 out of 25 unique DEGs were upregulated, while 15 genes were downregulated. Furthermore, the most common DEGs showed a similar tendency to vary in each of the treatment 
TABLE 3: DEGs classified into placenta and embryonic development of the GO biological process category.

\begin{tabular}{|c|c|c|}
\hline Group & Term & Genes \\
\hline \multirow{4}{*}{$\begin{array}{l}\text { Symbioflor } 1 \\
\text { group }\end{array}$} & $\begin{array}{c}\text { GO: 0001701 in utero embryonic } \\
\text { development }\end{array}$ & $\begin{array}{l}\text { MAFG, MAFF, XRCC2, GABPA, GNA12, SPINT1, EGLN1, BCL2L1, } \\
\text { MBNL1, ITGB1, TPM1, CITED2, HES1, NDEL1, MSX1, PSMC4, } \\
\text { HAND1, HSF1, TEAD4, PKD1, MKL2, LOC652826 (22) }\end{array}$ \\
\hline & GO: 0001890 placenta development & $\begin{array}{l}\text { VWF, PPARD, HSF1, HAND1, CCNF, PLCD3, SPINT1, HS6ST1, } \\
\text { EGLN1, PRDX3, CITED2 (11) }\end{array}$ \\
\hline & $\begin{array}{c}\text { GO: 0001892 embryonic placenta } \\
\text { development }\end{array}$ & HSF1, HAND1, SPINT1, EGLN1, CITED2 (5) \\
\hline & $\begin{array}{l}\text { GO: 0060136 embryonic process involved in } \\
\text { female pregnancy }\end{array}$ & HSF1, CITED2 (2) \\
\hline \multirow{6}{*}{ V583 group } & $\begin{array}{c}\text { GO: } 0043009 \sim \text { chordate embryonic } \\
\text { development }\end{array}$ & $\begin{array}{l}\text { GNA13, SYVN1, XRCC2, NDST1, EGLN1, BCL2L1, ITGB1, TPM1, } \\
\text { CITED2, HSF1, PKD1, MKL2, FLVCR1, BCL10, MAFF, ADAM10, } \\
\text { ZNF830, TGFBR2, SPINT1, MBNL1, LIG4, GAS1, CAPN2, BRCA1, } \\
\text { HES1, DLX2, TULP3, EP300, TSC1, PSMC4, SP3, HOXB6, MNX1, } \\
\text { TGIF1, RIPPLY1, FOXC1, APAF1, LOC652826, POFUT1 (39) }\end{array}$ \\
\hline & $\begin{array}{l}\text { GO: } 0009792 \sim \text { embryonic development } \\
\text { ending in birth or egg hatching }\end{array}$ & Same as GO: 0043009 chordate embryonic development (39) \\
\hline & $\begin{array}{c}\text { GO: 0001701 in utero embryonic } \\
\text { development }\end{array}$ & $\begin{array}{l}\text { GNA13, XRCC2, SYVN1, EGLN1, BCL2L1, TPM1, ITGB1, CITED2, } \\
\text { HSF1, PKD1, MKL2, FLVCR1, MAFF, ADAM10, ZNF830, SPINT1, LIG4, } \\
\text { MBNL1, CAPN2, HES1, TULP3, PSMC4, SP3, FOXC1, LOC652826 (25) }\end{array}$ \\
\hline & GO: 0001890 placenta development & $\begin{array}{l}\text { VWF, PPARD, CYP27B1, HSF1, SP3, CCNF, PLCD3, SPINT1, EGLN1, } \\
\text { CITED2 (10) }\end{array}$ \\
\hline & $\begin{array}{c}\text { GO: 0048701 embryonic cranial skeleton } \\
\text { morphogenesis }\end{array}$ & DLX2, TULP3, NDST1, TGFBR2, GAS1 (5) \\
\hline & $\begin{array}{l}\text { GO: 0060136 embryonic process involved in } \\
\text { female pregnancy }\end{array}$ & HSF1, SP3, CITED2 (3) \\
\hline
\end{tabular}

groups. However, the PPARD gene in particular differed from all other genes as it was upregulated in Symbioflor 1 and downregulated in V583.

\section{Discussion}

In our previous studies, we demonstrated the ability of E. faecalis OG1RF to translocate both intestinal and placental barriers and demonstrated the molecular mechanisms responsible for these actions by DNA microarray analysis $[7,8]$. However, the influence of E. faecalis in human pregnancy has not been fully elucidated, as different E. faecalis strains are expected to exhibit multiple roles. The objectives of the present study were to investigate and compare the different influences of putative pathogenic or probiotic E. faecalis on human placental trophoblast cells. Hence, two typical strains, representing the different lifestyles of this species, were evaluated for comparison: Symbioflor 1 and the pathogen V583. To the best of our knowledge, researchers have yet to investigate the influence of putative pathogenic or probiotic E. faecalis on human placental trophoblast cells by comparative transcriptomic analysis.

Our invasion assays demonstrated that both the pathogenic V583 strain and the probiotic Symbioflor 1 strain could adhere and internalize into human placental trophoblast cells. Furthermore, regardless of their diverse origins and lifestyles, these strains were found to show similar in vitro invasion ability. This observation was also consistent with our recent study of $E$. faecalis OG1RF in which $6.32 \pm 0.10 \log _{10} \mathrm{CFU} / \mathrm{mL}$ of bacteria were seen to invade and $2.23 \pm 0.29 \log _{10} \mathrm{CFU} / \mathrm{mL}$ were seen to internalize [8].
Similarly, Peng et al. found that the same three E. faecalis strains showed a similar adhesion rate but exhibited a different translocation rate in Ptk6 epithelial cell monolayers [15]. Bierne et al. reported that E. faecalis has the ability to internalize into intestinal LoVo cells [16]. These results demonstrated that different $E$. faecalis strains are able to pass through both intestinal and placental barriers, and their differences in invasion ability may be related to the cell model selected.

The influence of E. faecalis on human placental trophoblast cells was further investigated using DNA microarray analysis. We previously illustrated the biological effects and associated molecular mechanisms of E. faecalis OG1RF on placental function using a BeadChip microarray [8]. DNA microarrays have a distinct advantage over other techniques as they can provide both qualitative and quantitative data for a vast numbers of DEGs with high levels of sensitivity [17]. Li et al. used a DNA microarray to compare gene expression patterns between untreated and Aa-LPS-treated BeWo cells [18]. The changes in gene expression in human trophoblasts that pose a direct impact to placental and fetal health are now recognized as biomarkers [19]. According to the comparative results of DNA microarray analysis, the total number of DEGs appeared to be similar for both Symbioflor 1 and V583 treatment groups. Furthermore, the number of genes identified was slightly higher than that previously found in E. faecalis OGIRF [8]. However, almost half of the DEGs (both upregulated and downregulated) were unique to each group suggesting that different strains exhibit different effects on the placenta. According to our KEGG results, the V583 strain exhibited a greater number of signaling pathways than the Symbioflor 1 strain, thus indicating that pathogenic 


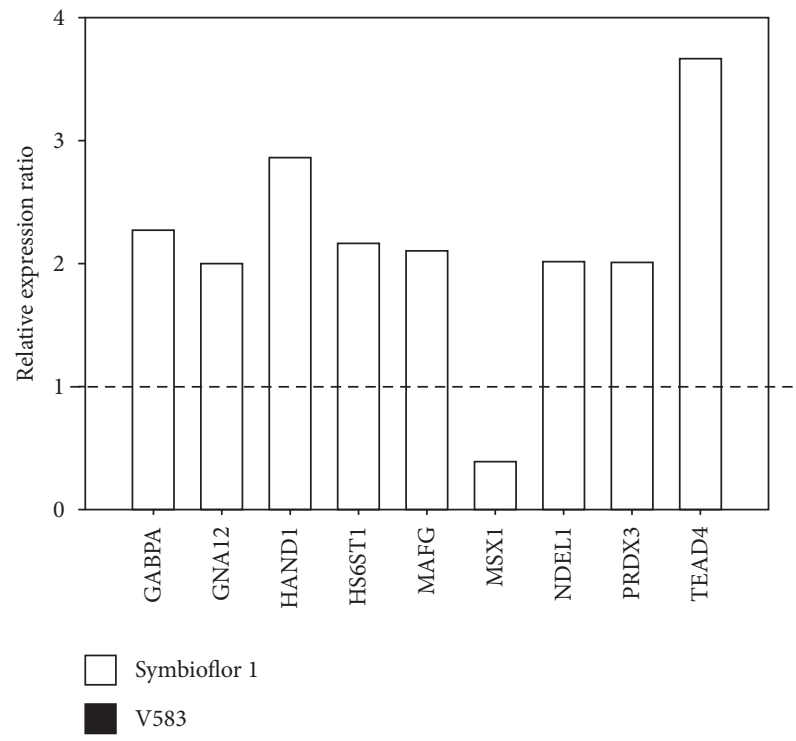

(a)

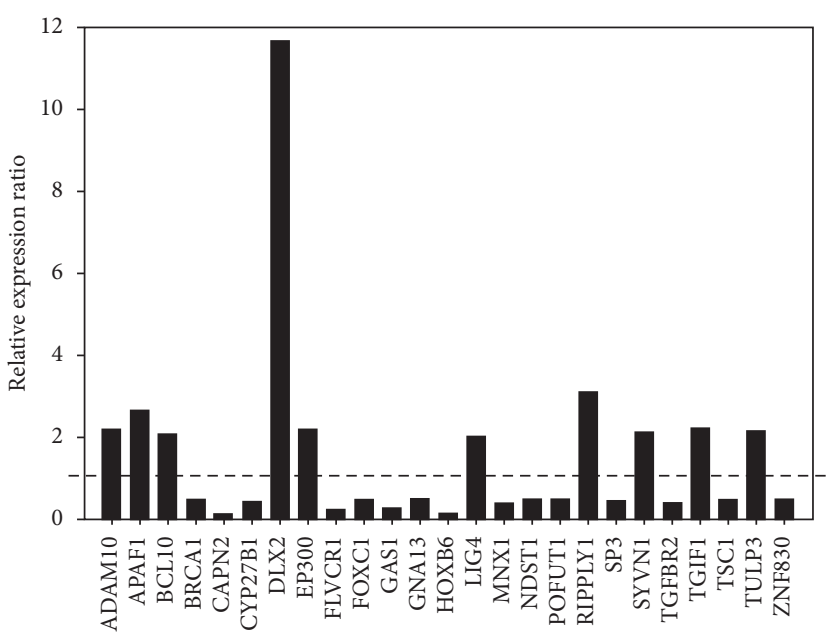

Symbioflor 1

V583

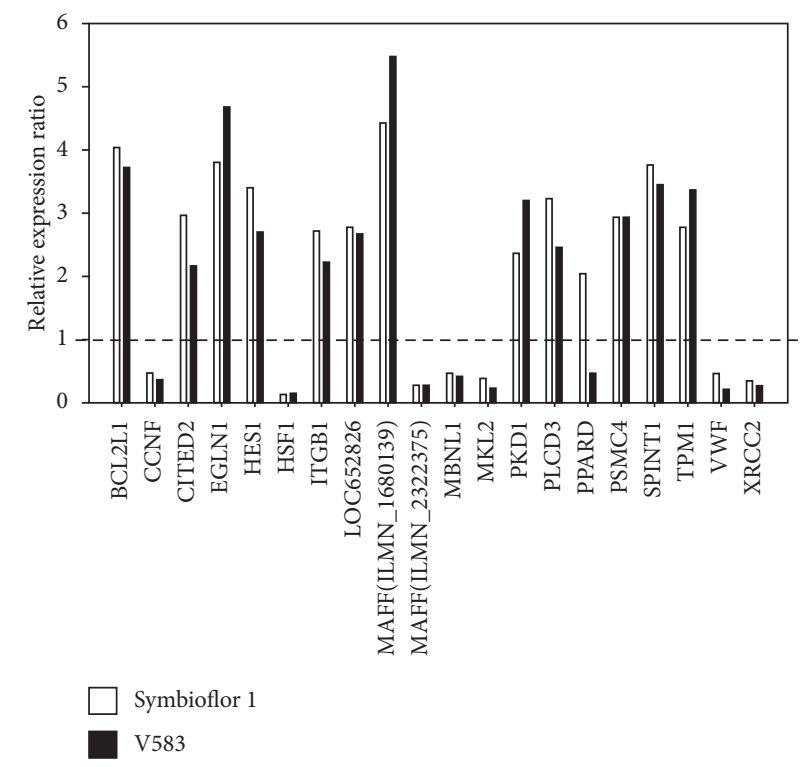

(b)

(c)

Figure 3: Fold change of DEGs classified into placenta and embryonic development. (a) Unique DEGs in the Symbioflor 1 treatment group; (b) unique DEGs in the V583 treatment group; (c) common DEGs in the Symbioflor 1 and V583 treatment groups. The relative expression ratio for each gene is presented in the histogram. A ratio greater than zero $(>1)$ indicates upregulation of gene expression, and a ratio below zero $(<1)$ indicates downregulation.

E. faecalis may in particular cause a stronger response in BeWo cells. Moreover, apoptosis, ErbB, B cell receptor, and leukocyte transendothelial migration signaling pathways were only activated in the V583 group. Similarly, pathogen-induced apoptosis in human placental trophoblasts can also lead to septicemia during pregnancy [18]. A previous study showed that the B cell receptor signaling pathway and leukocyte transendothelial migration were also found in the host cell infected by Streptococcus pneumoniae [20]. In particular, PPAR (peroxisome proliferator-activated receptor delta) is critically essential for placental development and function. This typical nuclear receptor has also been suggested to increase the placental fatty acid uptake [21]. Furthermore, studies have shown that PPAR- $\delta$-deficiency mice offspring can lead to growth retardation and impairment of neural development [22]. Our present results showed that PPARD was upregulated in the Symbioflor 1 treatment group but downregulated in the V583 treatment group. These results suggest that the presence of pathogenic E. faecalis may stunt fetal and placental growth and development. This result is in high agreement with our previous studies showing that pregnant mice given oral doses of E. faecalis OG1RF exhibit changes in terms of their fetal and placental growth and development $[7,8]$. Moreover, both ADAM10 and APAF1 
were exclusively upregulated in the V583 treatment group but not in the Symbioflor 1 treatment group. Previous studies have demonstrated that ADAM10 can mediate E-cadherin shedding and regulate epithelial cell-cell adhesion, thus exerting a direct impact on early embryonic development in vivo [23]. APAF1 is considered as a key player in apoptosis during embryonic development [24]. We also found that CAPN2, POFUT1, and GAS1 were all downregulated in the V583 treatment; these proteins have been shown to play an important role in embryonic development [25-27]. In particular, mouse embryos lacking Pofut1 have been shown to exhibit defects in their cardiovascular system [26]. Martinelli et al. found that Gas 1 mutant pups were only 3/4 the size of their control littermates [27] and showed similar abnormalities as fetal mice infected with E. faecalis OG1RF [7].

\section{Conclusions}

Our present analysis identified that a large proportion of transcriptional responses in BeWo cells differed when compared between infection caused by pathogenic and probiotic E. faecalis. Several unique DEGs and signal pathways were identified in the two strains. These data constitute a strong basis for understanding the mechanisms underlying the differential effects caused by pathogenic and probiotic strains of E. faecalis.

\section{Data Availability}

The datasets used and/or analyzed during the current study are available from the corresponding author upon request.

\section{Conflicts of Interest}

The authors declare that they have no conflicts of interest.

\section{Acknowledgments}

This research project was supported by the National Natural Science Foundation of China (31570075 and 31770133), Health Young and Middle-aged Backbone Personnel Training Project of Fujian Provincial Health Commision (2019-ZQNB-21), Foundation for Program of Department of Education from Fujian Province (JAT190849), and Research Program of Xiamen Medical College (K2015-09). The authors thank EditSprings for linguistic assistance during the preparation of this manuscript.

\section{Supplementary Materials}

Table S1. Differential gene expression profile of BeWo cell in response to Enterococcus faecalis Symbioflor 1 invasion. Table S2. Differential gene expression profile of BeWo cell in response to Enterococcus faecalis V583 invasion. Table S3. Differentially expressed genes affected by Enterococcus faecalis Symbioflor 1 classified with GO database. DEGs were characterized functionally by comparison against GO database and classified into three different categories, namely, the biological process, cellular component, and molecular function. Table S4. Differentially expressed genes affected by Enterococcus faecalis V583 classified with GO database. DEGs were characterized functionally by comparison against GO database and classified into three different categories, namely, the biological process, cellular component, and molecular function. (Supplementary Materials)

\section{References}

[1] O. Ben Braïek and S. Smaoui, "Enterococci: between emerging pathogens and potential probiotics," BioMed Research International, vol. 2019, Article ID 5938210, 13 pages, 2019.

[2] O. Baccouri, A. M. Boukerb, L. B. Farhat et al., "Probiotic potential and safety evaluation of Enterococcus faecalis OB14 and OB15, isolated from traditional Tunisian Testouri cheese and Rigouta, using physiological and genomic analysis," Frontiers in Microbiology, vol. 10, p. 881, 2019.

[3] M. S. Gilmore, F. Lebreton, and W. van Schaik, "Genomic transition of enterococci from gut commensals to leading causes of multidrug-resistant hospital infection in the antibiotic era," Current Opinion in Microbiology, vol. 16, no. 1, pp. 10-16, 2013.

[4] K. Shigematsu, A. Asai, M. Kobayashi, D. N. Herndon, and F. Suzuki, "Enterococcus faecalis translocation in mice with severe burn injury: a pathogenic role of CCL2 and alternatively activated macrophages (M2aM $\phi$ and $\mathrm{M} 2 \mathrm{cM} \phi)$," Journal of Leukocyte Biology, vol. 86, no. 4, pp. 999-1005, 2009.

[5] N. Steck, M. Hoffmann, I. G. Sava et al., "Enterococcus faecalis metalloprotease compromises epithelial barrier and contributes to intestinal inflammation," Gastroenterology, vol. 141, no. 3, pp. 959-971, 2011.

[6] R. L. Kim and C. Thompson, "The infectious origins of stillbirth," American Journal of Obstetrics and Gynecology, vol. 189, no. 3, pp. 861-873, 2003.

[7] Q. Tan, H. Xu, F. Xu et al., "Survival, distribution, and translocation of Enterococcus faecalis and implications for pregnant mice," FEMS Microbiology Letters, vol. 349, no. 1, pp. 32-39, 2013.

[8] Q. Tan, F. Xu, H. Xu, L. Qiu, X. Tao, and H. Wei, "Transcriptomic profiling of human placental trophoblasts in response to infection with Enterococcus faecalis," Journal of Food Quality.vol. 2018, Article ID 5607641, 11 pages, 2018.

[9] E. Jiménez, M. L. Marín, R. Martín et al., "Is meconium from healthy newborns actually sterile?" Research in Microbiology, vol. 159, no. 3, pp. 187-193, 2008.

[10] R. Odriozola, M. A. Ehrmann, M. Korakli, S. Yazaji, and R. F. Vogel, "Phenotypic and genotypic analyses of lactic acid bacteria in local fermented food, breast milk and faeces of mothers and their babies," Systematic and Applied Microbiology, vol. 34, no. 2, pp. 148-155, 2011.

[11] Y. Tao, F. Huang, Z. Zhang et al., "Probiotic Enterococcus faecalis Symbioflor 1 ameliorates pathobiont-induced miscarriage through bacterial antagonism and Th1-Th2 modulation in pregnant mice," Applied Microbiology and Biotechnology, vol. 104, no. 12, pp. 5493-5504, 2020.

[12] H. C. Tao, M. Solheim, L. Snipen, I. F. Nes, and D. A. Brede, "Comparative genomic analysis of pathogenic and probiotic Enterococcus faecalis isolates, and their transcriptional responses to growth in human urine," PLoS One, vol. 5, no. 8, Article ID e12489, 2010.

[13] H. Tang, Z. Jiang, H. He et al., "Uptake and transport of pullulan acetate nanoparticles in the BeWo b30 placental barrier cell model," International Journal of Nanomedicine, vol. 13, pp. 4073-4082, 2018. 
[14] C. Li, N. J. Bokil, M. Totsika et al., "The co-transcriptome of uropathogenic E scherichia coli -infected mouse macrophages reveals new insights into host-pathogen interactions," Cellular Microbiology, vol. 17, no. 5, pp. 730-746, 2015.

[15] Z. Kakkanat, V. Krey, H. Wei et al., "Impact of actin on adhesion and translocation of Enterococcus faecalis," Archives of Microbiology, vol. 196, no. 2, pp. 109-117, 2014.

[16] H. Tan, L. Travier, T. Mahlakõiv et al., "Activation of type III interferon genes by pathogenic bacteria in infected epithelial cells and mouse placenta," PLoS One, vol. 7, no. 6, Article ID e39080, 2012.

[17] I. S. Segundo-Val and C. S. Sanz-Lozano, "Introduction to the gene expression analysis," Methods in Molecular Biology, vol. 1434, pp. 29-43, 2016.

[18] Y. Li, Y. Shibata, L. Zhang, N. Kuboyama, and Y. Abiko, "Periodontal pathogen Aggregatibacter actinomycetemcomitans LPS induces mitochondria-dependent-apoptosis in human placental trophoblasts," Placenta, vol. 32, no. 1, pp. 11-19, 2011.

[19] C.-R. Roh, V. Budhraja, H.-S. Kim, D. M. Nelson, and Y. Sadovsky, "Microarray-based identification of differentially expressed genes in hypoxic term human trophoblasts and in placental villi of pregnancies with growth restricted fetuses," Placenta, vol. 26, no. 4, pp. 319-328, 2005.

[20] M. S. Jonczyk, L. Escudero, N. Sylvius, M. Norman, B. Henriques-Normark, and P. W. Andrew, "Variation in inflammatory response during pneumococcal infection is influenced by host-pathogen interactions but associated with animal survival," Infection and Immunity, vol. 84, no. 4, pp. 894-905, 2016.

[21] Y. Xu, Q. Wang, T. J. Cook, and G. T. Knipp, "Effect of placental fatty acid metabolism and regulation by peroxisome proliferator activated receptor on pregnancy and fetal outcomes," Journal of Pharmaceutical Sciences, vol. 96, no. 10, pp. 2582-2606, 2007.

[22] A. Jawerbaum and E. Capobianco, "Review: effects of PPAR activation in the placenta and the fetus: implications in maternal diabetes," Placenta, vol. 32, no. 2, pp. S212-S217, 2011.

[23] T. Maretzky, K. Reiss, A. Ludwig et al., "ADAM10 mediates E-cadherin shedding and regulates epithelial cell-cell adhesion, migration, and -catenin translocation," Proceedings of the National Academy of Sciences, vol. 102, no. 26, pp. 9182-9187, 2005.

[24] D. Buchholz, E. Maiani, and F. Ceccon, "Apaf1 in embryonic development - shaping life by death, and more," The International Journal of Developmental Biology, vol. 59, no. 1, pp. 33-39, 2015.

[25] F. Raynaud, A. Marcilhac, K. Chebli, Y. Benyamin, and M. Rossel, "Calpain 2 expression pattern and sub-cellular localization during mouse embryogenesis," The International Journal of Developmental Biology, vol. 52, no. 4, pp. 383-388, 2008.

[26] Y. Okamura and Y. Saga, "Pofut1 is required for the proper localization of the Notch receptor during mouse development," Mechanisms of Development, vol. 125, no. 8, pp. 663-673, 2008.

[27] D. C. Martinelli and C.-M. Fan, "The role of Gas1 in embryonic development and its implications for human disease," Cell Cycle, vol. 6, no. 21, pp. 2650-2655, 2007. 